\title{
NARRATION ET LANGAGE DANS L'OEUVRE DE RAYMOND QUENEAU, ZAZIE DANS LE METRO
}

\section{INTRODUCTION}

Zazie dans le métro est un roman de Raymond Queneau, paru en 1959 et traduit dans plusieurs langues dont le slovène, adapté au théâtre plusieurs fois et, au cinéma, en octobre 1960, par Louis Malle. En 1966, Jacques Carelman fait même de Zazie dans le métro une bande dessinée. En 19 chapitres, Queneau nous retrace les aventures non seulement du personnage éponyme, mais d'autres encore, qui se laissent guider par leur destinée, leurs choix, leurs décisions... et surtout par l'art de l'auteur dans les domaines de la narration, de la parodie, des jeux de langage... Son texte est complexe et l'écriture soignée. Queneau maîtrise différents niveaux de langue : on assiste à un va-et-vient incessant entre le populaire et le soutenu. C'est là également que réside la force comique de l'oeuvre. Cet article se propose donc d'étudier brièvement la matière de l'oeuvre narrative de Raymond Queneau, Zazie dans le métro.

\section{NARRATION, NARRATEUR ET FOCALISATION}

L'histoire de Zazie dans le métro se déroule au cours du $\mathrm{XX}^{\mathrm{e}}$ siècle. Dans les années cinquante, Jeanne Lalochère, couturière à Saint-Montron envoie à Paris pour la fin de semaine, chez son frère, Gabriel, sa fille, Zazie, qui a entre dix et treize ans. Zazie voudrait surtout découvrir le métro, mais dès son arrivée, la grève du métro la démoralise. La fillette va pourtant parcourir la capitale. Il lui arrive un certain nombre d'aventures liées à son caractère indépendant et à son franc-parler. Elle démarre ses aventures seule ou accompagnée de personnages assez bizarres - son oncle Gabriel, dit « Tonton Gabriel », le policier Trouscaillon, le chauffeur de taxi Charles, Madame veuve Mouaque, le guide touristique slave Fédor Balanovitch, les touristes multinationaux, etc. Zazie visite d'abord le marché aux puces, monte ensuite sur la tour Eiffel, apprécie le numéro de Gabriel au Mont de Piété, etc. Son aventure se déroule ainsi jusqu'à ce qu'elle participe à une bagarre dans un café-restaurant, où les protagonistes s'échappent au petit matin, au moment où le métro se remet en marche. À sa mère qui l'interroge sur son séjour, elle répond, et ce sont les derniers mots du roman : "J'ai vieilli ».

L'histoire de Zazie dans le métro est fictive. Il s'agit d'un récit " classique ", raconté à la troisième personne par un narrateur qui n'est pas un personnage du récit. La narration est postérieure aux événements racontés et l'ordre temporel du

\footnotetext{
*Adresse de l'auteur : Filozofska fakulteta, Oddelek za prevajalstvo, Aškerčeva 2, 1000 Ljubljana, Slovénie. Mél: sonia.vaupot@guest.arnes.si
} 
récit coïncide avec l'ordre chronologique de l'histoire. La distance entre la narration (ultérieure) et l'histoire est marquée par l'emploi des temps du passé et du présent de narration. Il est intéressant de noter que le passé simple, signe par excellence du récit écrit, est engagé dès la première phrase: "Doukipudonktan, se demanda Gabriel excédé ».

Bien que le narrateur soit invisible au niveau de l'histoire, on sent qu'il est omniprésent, car il est le seul qui ouvre accès aux impressions des personnages. Mais, le narrateur n'est pas omniscient, ce que nous démontrent les réserves et les lacunes qui concernent les personnages romanesques : « le ptit type » du début du roman est " probablement " le mari de la dame allergique à Barbouze. Le narrateur est un observateur externe qui interprète les comportements et traduit les points de vue des personnages laissant parfois le lecteur dans l'incertitude. Par exemple, lorsque Gabriel désigne le prétendu Panthéon, le narrateur se contente d'un vague "désigne...quelque chose " (p. 14). De même, le narrateur néglige ce que pouvait faire Marceline quand Zazie s'est enfuie. L'information donnée au lecteur comporte ainsi des lacunes. On ne sait rien des propos que Zazie prête à Turandot (p. 34). En outre, Queneau ne fera qu'une seule allusion au lien familial qui unit Gabriel et Charles (p. 15), et la transformation d'un " pote » (p. 13) en « beau-frère » (p. 15) est peu claire.

En rapportant les pensées de ses personnages, le narrateur passe souvent d'un langage soutenu à des expressions faisant partie du monologue intérieur du personnage. Par exemple, «ça va sûrement jeter le désarroi » (p. 32) exprime la pensée du narrateur qui garde une distance face à Zazie, contrairement à la phrase qui apparaît plus loin : « elle finit par se déclarer que c'est drôlement con les contes de fées ».

L'ensemble du commentaire narratif est marqué par une proximité avec les personnages sur le plan langagier, et une mise à distance des protagonistes comme l'indiquent les notations qui suivent les répliques dans les dialogues (par exemple : " répondit Gabriel tombant dans le piège », p. 23). Les innombrables va-et-vient et points de vue du narrateur et de ses personnages s'insèrent donc tout au long du roman sans toutefois interrompre le récit.

\section{TEMPORALITÉ ET DÉFOCALISATION}

L'action du roman se déroule selon une temporalité linéaire. Trente-six heures environ s'écoulent, réparties sur trois jours ou deux jours et une nuit, entre l'arrivée et le départ des Lalochère : "Alors, je vous retrouve ici après-demain (p.12) ; Elle reste que deux-trois jours, dit Charles (p.21); L'objet qui l'avait tant occupée pendant un jour et deux nuits (p. 188)».

Les deux premiers chapitres racontent le premier contact de Zazie avec son oncle et la capitale (le premier jour). L'essentiel de l'aventure est vécu le lendemain. L'épilogue relate les événements advenus le troisième jour.

L'action du récit est balisée par quelques événements. Gabriel part chercher Zazie à la gare d'Austerlitz l'après-midi du premier jour, et Charles se joint à eux « pour prendre l'apéro » (p. 17) et dîner chez Gabriel (p. 18-22). Le lendemain matin, Zazie reste dans «les vécés» (p. 32) durant « un bon quart d'heure » avant de quitter 
le domicile de son oncle. Sa fugue dure le restant de la matinée. Elle retourne chez Gabriel un peu avant midi, à l'heure où les " midineurs » munis de leur gamelle se pressent chez Turandot (p.71). Seul le cordonnier Gridoux prend son repas « après le coup de feu, à l'environ d'une heure » (p. 73). L'après-midi, Zazie, Gabriel et Charles visitent la tour Eiffel. Plus tard, ils rencontrent la veuve Mouaque qui leur indique l'heure : "seize heures quinze, dit la bourgeoise » (p. 100). Or quelque temps plus tard, Trouscaillon, la veuve Mouaque, le Sanctimontronais et Zazie entendent sonner " quatre heures... au clocher d'une église voisine » (p. 114). Enfin, plus aucune indication temporelle n'apparait dans la suite du texte avant le dernier chapitre. S'enchaînent les parties de billard et de pimpon, le dîner au Sphéroïde, la représentation au Mont-de-Piété, la dégustation de soupe à l'oignon et la bagarre finale aux Nyctalopes. Mais au matin du troisième jour, le temps des horloges réapparait : 《 il était six heures passées » (p. 188). "À la demie » (p. 189), Jeanne est à la gare d'Austerlitz pour prendre le train de « six heures soixante » en compagnie de Zazie.

Les diverses actions vécues ou perçues par les personnages dépendent ainsi d'un ordre chronologique et logique. On note que l'axe temporel de trente-six heures autour duquel s'organise le récit est parfois effacé. Par exemple, le récit couvrant le déplacement entre le « bistrot du coin » et la Cave est absent (entre les chap. 1 et 2). De même, peu d'informations nous sont données sur la première nuit vécue par les personnages (entre les chap. 2 et 3). Queneau a aussi recours à l'ellipse : il ne dit rien sur la dernière étape du trajet de la gare à la Cave, il ménage le suspense à propos de l'activité professionnelle de Gabriel, etc.

La narration se détourne parfois du protagoniste Zazie. Pourtant, si le fil logique de la narration est interrompu par des bifurcations du récit, l'axe temporel continue à se déployer.

D'un point de vue plus général, les chapitres 12 à 16 se succèdent et se recoupent en partie. L'aventure se dédouble dans le cours du roman. Deux actions simultanées apparaissent dans les chapitres 3 et 4 d'une part, 11 et 12 d'autre part. Puis, l'histoire de Gabriel qui est l'« archiguide » d'un groupe à travers Paris (chap. 12, 14 et 16) alterne avec la description de scènes situées à la Cave (chap. 13) ou deux étages au-dessus (chap. 13 et 15). Ainsi, l'action progresse même si le récit est entrecoupé par d'autres personnages que Zazie. Ces atteintes à l'ordre linéaire ordonnent les événements du récit et en dessinent les contours. Dans les chapitres 3 et 4 , le narrateur met en évidence le fait que le protagoniste est Zazie (chap. 4), mais que le personnage de Gabriel est tout aussi essentiel (chap. 3).

Cette brève étude fait ressortir que les quelques ellipses, dédoublements de la temporalité de l'action et défocalisation par rapport au personnage principal, ne bousculent pas non plus l'ordre linéaire du récit. Chaque chapitre correspond à une unité narrative, qui dépend de la précédente et de la suivante. À l'intérieur des épisodes n'apparaît aucune brisure ou retour en arrière systématique. Par ailleurs, on ne sait rien de Saint-Montron, un mot réduit à la simple connotation de bled ou patelin, et le récit de Zazie au chapitre 5 nous livre ses désirs immédiats plutôt qu'un aperçu de son passé. Aussi, Zazie se retrouve sur le quai de gare d'où elle était partie sans que rien n'ait changé en quarante-huit heures. 


\section{LE CHOIX DU LANGAGE}

L'oeuvre romanesque de Queneau est souvent placée sous le signe de l'anti-langage. Roland Barthes (1964 : 125-131) remarque dans l'article "Zazie et la littérature " que le langage est au centre de l'entreprise romanesque de Queneau. L'auteur est considéré, notamment avec le personnage de Zazie, comme l'inventeur d'un nouveau langage.

Cette langue nouvelle est l'image même du langage populaire de cette époque. Queneau exploite les ressources langagières de l'argot ou du " néo-français ", langage parlé devenant langage écrit au moyen de diverses ressources langagières. En reprenant le " roman parlé », le romancier donne l'illusion à travers son langage que le récit est écrit dans la langue des personnages. Pour cela, il utilise à profusion les calembours, inversions, répétitions, mots-valises.... Ces procédés donnent au roman un style particulier et mettent à jour un monde artificiel ou irréel.

Queneau adapte ainsi le langage écrit à la langue parlée. Il se livre entre autres à des néologismes : "Zazie » a été créé par dérivation du nom "zazous » donné aux jeunes gens qui, au temps de l'Occupation et dans les années qui suivirent, se signalaient par leur mode et leur passion du jazz. Les personnages de Queneau se caractérisent d'abord par le langage particulier qu'ils emploient : « du boudin zaricos verts que vzavez jamais zétés foutus de fabriquer. (p.132)»

Par ailleurs, Queneau narre une succession de faits qui s'enchaînent. Son texte est caractérisé par une segmentation de l'écrit et une majorité de dialogues. Les évocations du passé sont entre autres racontées par les personnages lors de dialogues. La logique du récit correspond parfois à celle de la pensée arbitraire des personnages qui se déploie pendant la conversation. Ainsi, les dialogues sont en majorité chargés d'irrégularités et présentent une tendance à l'économie phonétique et syntaxique. Décrivant la vie quotidienne de la population à cette époque, Queneau transgresse ainsi volontairement les règles d'orthographe, graphie et pratique phonétiques de la langue française (l'« Ortograf fonétik »). Outre la phrase d'attaque : "Doukipudonktan », le roman contient de nombreuses expressions affranchies de toute règle d'orthographe telle que "Skeutadittaleur " (p. 10), «Lagoçamilébou » (p. 37) ou encore " a boujpludutou » et " bloudjiins » (p. 47), etc. De plus, l'oeuvre présente une mise en relief des erreurs qui sont courantes dans le code parlé relâché ou des éléments peu perceptibles à l'oral : les fautes de liaison « le boudin zaricos verts » (p. 132) ; l'absence de liaison " « c'est hun cacocalo » (p. 18); la syncope ou élision du - e - "ptite mère » (p.10) et «j'veux pas » (p. 18); la disparition des consonnes « autt chose» (p. 18); la chute du - ne " ça m'étonne pas « p. 9 » et des pronoms personnels « isra » (p. 49) ; la transformation nominale " meussieu » (p. 9)... Il reprend également la syntaxe du français populaire « qu'il dit Gabriel » (p. 10) ou encore « comme vous que j'vous dis $\gg($ p. 74).

Or malgré l'apparence ludique de "l'ortografe fonétik », l'écriture de Queneau reste fondée sur la réalité du phonétisme français actuel, comme le montre la scène suivante (p. 163) : 
- M'autorisez-vous donc à de nouveau formuler la proposition interrogative qu'il y a quelques instants j'énonça devant vous?

- J'énonçai, dit l'obscur.

- J'énonçais, dit Trouscaillon.

- J'énonçai sans esse.

Cette querelle entre les deux personnages porte sur l'opposition E fermé/E ouvert du passé simple et de l'imparfait, souvent négligée dans la prononciation populaire. Les archaïsmes « mainte » (p.30) et l'usage, même fautif, du passé simple (« fermit les yeux » (p. 66), « jamais on upu croire qu'il y en u tant» (p.179) ) ou du subjonctif imparfait (« étonné que le costaud répliquât » (p.10)) offrent l'apparence d'un autre registre, plus soutenu, mais mettent surtout en valeur la variété des jeux et des écarts langagiers de Queneau.

Aussi, Queneau (1950) semble avoir mis en pratique la théorie, exposée dans son article Écrit en 1937, qui se réfère au Langage de Vendryes. Il considère en effet que le français parlé est un langage très différent du français écrit et réalise que le français moderne devait se dégager des conventions de l'écriture qui l'enserrent encore (conventions tant de style que d'orthographe et de vocabulaire). Ce qui était le plus important pour passer du français écrit ancien, d'une langue morte, à un français moderne écrit, correspond selon lui à la langue réellement parlée. Queneau soutient l'adoption d'une orthographe phonétique comme source d'une nouvelle littérature et souligne la prééminence de l'oral sur l'écrit.

En usant de ces procédés, Queneau transgresse volontairement les normes. Le développement de l'histoire prend une dimension déroutante et certains éléments apparaissent comme fantaisistes ou démesurés (p. 179) :

C'était maintenant des troupeaux de loufiats qui surgissaient de toutes parts. Jamais on upu croire qu'il y en u tant. Ils sortaient des cuisines, des caves, des offices, des soutes. Leur masse serrée absorba Gridoux puis Turandot aventuré parmi eux. Mais ils n'arrivaient pas à réduire Gabriel aussi facilement. Tel le coléoptère attaqué par une colonne myrmidonne, tel le bœuf assailli par un banc hirudinaire, Gabriel se secouait, s'ébrouait, s'ébattait, projetant dans des directions variées des projectiles humains qui s'en allaient briser tables et chaises ou rouler entre les pieds des clients.

Cependant, malgré des éléments fantaisistes et même si le roman ne cesse jamais, du premier mot au dernier dialogue, de jouer sur les mots et le langage, Queneau affiche un souci de réalisme tout au long du roman par des allusions à la vie quotidienne et notamment au métro. 


\section{CONCLUSION}

Zazie est une adolescente curieuse et insolente qu'on a souvent comparée à Lolita (Braffort 2003). Elle utilise un langage très familier. Au fil du récit, elle mûrit au contact des adultes, s'interroge et pose bien des questions sur son entourage et la société en général.

Pour sa part, Queneau réalise une sorte de chronique parisienne des années 50. Mais, le monde romanesque est mis en question à mesure qu'il se construit. Ainsi, le récit se présente aussi comme une construction artificielle dont la facticité est signalée par l'auteur lui-même. Vers la fin de sa vie, Queneau reviendra sur l'idée de " néo-français " constatant le rôle de standardisation, de la radio et des médias de masse. D'ailleurs, on retrouve des allusions à la presse dans Zazie dans le métro dont l'espace romanesque intègre quelques quotidiens à l'usage des classes moyennes, dénonçant ainsi l'attachement de Queneau pour les systèmes de communication et les vecteurs d'information.

\section{Bibliographie}

Queneau, Raymond (1994) Zazie dans le métro. Paris : Gallimard.

BrafForT, Paul (2003) « Les quatre petites filles. » Europe, 152-163.

BARTHES, Roland (1964) « Zazie et la littérature. » Essais critiques, 125-131.

Bigot, Michel (1994) Zazie dans le métro de Raymond Queneau. Paris : Gallimard.

Blanchon, Jean A. (1991) "L'Attribut en question : Super-Zazie dans le métro. » L'Information Grammaticale, 27-29.

Bourdette Donon, Marcel (2001) Raymond Queneau : l'œeil, l'oreille et la raison. Paris : L'Harmattan.

CAtonné, Jean-Marie (1992) Queneau. Paris : Les Dossiers Belfond.

DAVID, Pierre (1994) Dictionnaire des personnages de Raymond Queneau. Limoges : Pulim.

Diament, Henri (1990) «Onomastique fiction: Science et fantaisie dans deux romans de Raymond Queneau. » Nouvelle Revue d'Onomastique, 215-223.

Jullien, Dominique (2000) « Zazie dans la brousse. » Romanic Review, 263-278.

LeCUREUR, Raymond (2002) Raymond Queneau. Paris : Les Belles Lettres.

Martin, Jean Pierre (1994) " Raymond Queneau : Le Roman à voix basse. " Poétique, 291-300.

MARTINET, André (1955) Économie des changements phonétiques. Bern : Francke.

MARTinet, André (1975) Évolution des langues et reconstruction, Paris : Presses Universitaires de France.

QueNEAU, [Raymond] (1950) Bâtons, chiffres et lettres, Paris : Gallimard.

Queval, Jean (1984) Raymond Queneau, portrait d'un poète. Paris : H. Veyrier.

Raymond Queneau. Europe - revue littéraire mensuelle, 2003.

REDFERN, Walter. D. (1980) Queneau : Zazie dans le métro. London : Grant \& Culter. Souchier, Emmanuël (1991) Raymond Queneau. Paris : Ed. du Seuil. 


\section{Povzetek \\ NARACIJA IN JEZIK V QUENEAUJEVEM DELU CICA V METROJU}

Cica v metroju, roman Raymonda Queneauja iz leta 1959, je bil preveden v več jezikov, tudi slovenščino. V 19 poglavjih nam avtor opisuje dogodivščine naslovne, pa tudi drugih oseb. Besedilo je kompleksno, pisava izpiljena. Avtor obvladuje več jezikovnih ravni. Priča smo prehajanju med pogovornim in knjižnim jezikom, in tudi v tem se kaže pripovedna moč romana. V članku je predstavljena kratka študija narativnih posebnosti v Queneaujevem delu.

Zgodba o Cici je izmišljena, predstavljena s stališča pripovedovalca. Gre za klasično pripoved, kjer pripovedovalec ni del zgodbe, je pa v pripovedi vseskozi prisoten. Osebe, njihove lastnosti in dejanja so predstavljeni $\mathrm{z}$ gledišča pripovedovalca, tako da bralec o dogajanju nikdar ne dobi »popolne« slike. Dogajanje v romanu sledi linearnemu kronološkemu razporedu dogodkov zgodbe, čeprav se časovni vidik občasno zabriše. Približno 36 ur poteče od začetka do konca zgodbe. Posamezne dele pripovedi označujejo in zaznamujejo nekateri ključni dogodki. Vendar niti elipse in podvajanja niti defokalizacije, povezane z glavno osebo, ne posežejo v linearnost zgodbe.

Za romane Queneauja pogosto velja, da so pisani v »protijeziku«. Avtor v njih pravzaprav prilagaja pisni jezik govorjenemu jeziku. Sledeč »govorjenemu romanu«, ustvarja iluzijo, da je zgodba napisana $v$ jeziku osebe iz romana. Za sam tekst je značilna segmentacija zgodbe in prevlada dialogov. Kljub vsemu pa avtor skozi ves roman z aluzijami na vsakdanje življenje, predvsem seveda na metro, poskrbi, da se ohrani realističnost pisave. 